\title{
Avascular Necrosis of the Hip in Primary
} Care

\author{
Nurul Nadia Baharum ${ }^{1, *}$, Farnaza Ariffin ${ }^{1}$, Mohammad Hanafiah², Shahrul Hisham Sulaiman ${ }^{3}$ \\ 'Primary Care Medicine, Faculty of Medicine, Universiti Teknologi MARA, Selayang Campus, Batu Caves, Malaysia \\ ${ }^{2}$ Department of Radiology, Faculty of Medicine, University Teknologi MARA, Sungai Buloh Campus, Sungai Buloh, Malaysia \\ ${ }^{3}$ Department of Orthopaedics, Faculty of Medicine, University Teknologi MARA, Sungai Buloh Campus, Sungai Buloh, Malaysia
}

Avascular necrosis, or osteonecrosis of the femoral head, is a debilitating condition which leads to the destruction of the hip joint due to an interruption in the blood supply to the bony region and is most commonly due to trauma. The case discussed here has been highlighted as it presented as non-traumatic osteonecrosis of the femoral head with an absence of risk factors in a healthy adult male. A 37-year-old male presented with a 4-month history of recurrent left hip pain, which worsened with initiation of movement and weightbearing on the affected side. The patient was overweight but normotensive with a full range of movement of the hips bilaterally. There were no abnormalities detected on initial X-ray images of the left hip. However, due to the persistent pain and sclerotic changes in a subsequent X-ray, a magnetic resonance image of the bilateral hips was obtained, leading to the diagnosis of osteonecrosis of the bilateral femoral heads. Due to the lack of improvement with physiotherapy and analgesia, the patient was subjected to conservative surgery of the symptomatic left hip with concurring evidence of avascular necrosis based on intraoperative anatomical biopsy. This case emphasizes the importance of identifying underlying issues during history taking and physical examination in adults without risk factors. The early diagnosis of osteonecrosis assists in preventing joint collapse and can delay the requirement of joint replacements. High levels of suspicion are necessary to instigate investigation in persistent cases without the presence of risk factors.

Keywords: Osteonecrosis; Femur Head Necrosis; Risk Factors; Early Diagnosis

Received: March 23, 2019, Revised: April 24, 2019, Accepted: June 9, 2019

*Corresponding Author: Nurul Nadia Baharum https://orcid.org/0000-0001-8922-1296

Tel: +60-3-6126-6000, Fax: +60-3-6126-4888, E-mail: 2014525855@isiswa.uitm.edu.my 


\section{INTRODUCTION}

Osteonecrosis, also known as avascular necrosis, aseptic necrosis, or ischemic necrosis, is a pathology that can progress to the permanent destruction of bones. Clinically, osteonecrosis of the femoral head (ONFH) can be classified by the presence or absence of trauma. Traumatic causes of osteonecrosis of femoral head have been studied extensively and the pathophysiology is well understood. In these cases, trauma leads to the interruption of the vascular supply of the femoral head. ${ }^{1)}$ Meanwhile, non-traumatic osteonecrosis of the femoral head (NONFH) refers to the death of bone tissue due to an interruption of the blood supply to the bone with the clear absence of trauma. The causes of NONFH are not clearly understood, but cases have been associated with corticosteroid use, alcoholism, and systemic medical conditions such as thrombophilia, sickle cell disease, systemic lupus erythematosus and in younger patients, Legg-Calve-Perthes disease, and slipped capital femoral epiphysis. ${ }^{1)}$ This case highlights a case of NONFH with the absence of associated risk factors.

\section{CASE REPORT}

A 37-years-old male presented to UiTM Primary Care Clinic, Sungai Buloh, Selangor, Malaysia with a history of persistent left hip pain. The patient had underlying bronchial asthma, present since childhood, that had been well-controlled with salbutamol use as required. The patient was not on any inhaled corticosteroids.

The patient first presented with a 2-week history of left anterior hip pain that occurred after he slipped on the stairs and landed on his left knee with no direct trauma to the hip. The pain worsened with initiation of movement and weightbearing on the affected joint, especially when exiting a car or climbing stairs. He was overweight, normotensive, with full range of movement of the bilateral hips. The patient had no history of prolonged corticosteroid use, alcoholism, and he had no systemic medical conditions. Left hip X-ray showed no obvious evidence of fracture and he was discharged with analgesia. It was difficult to attribute the fall as a cause for the hip pain since there was no direct trauma to the hip; however, the hip pain could be a referred pain from the knee.

The patient then presented a month later complaining of acute lower back pain which radiated to the left groin and had been present for 3 days. He was noted to have an antalgic gait with paravertebral muscle spasm. On examination, the straight leg raise test was negative with normal neurological examination of the lower limbs. Lumbosacral Xray was normal and the patient was discharged with analgesia. He was reviewed 2 days later, stating improvement and no longer had an antalgic gait.

Subsequently, the patient continued to have several recurrent episodes of intermittent left hip pain. In between attacks, his pain would partially subside. He returned to the clinic with similar symptoms and this prompted the primary care team to arrange for an orthopedic assessment. On examination, the patient had limited internal rotation and external rotation of his left hip and a repeated left hip X-ray showed sclerotic changes (Figure 1). In view of these findings, a magnetic resonance image (MRI) of bilateral hip joints was arranged (Figure 2). The results revealed bilateral femoral head avascular necrosis with left hip synovitis. This was classified as Association Research Circulation Osseous stage II.

The patient underwent core decompression of the left hip and an autologous iliac bone graft around 5 months after his first point of care assessment. His right hip was treated conservatively as it was asymptomatic and the changes from the MRI were minimal. Histopathological examination of the left femoral head biopsy showed necrotic bony tissue with no malignancy features seen (Figure 3). The patient was discharged on oral paracetamol and tramadol and was put on strict non-weight bearing crutches.

The patient attended regular follow-up appointments following the surgery and his progress was monitored according to pain control and hip X-ray examinations. In general, his pain score was mild, and his serial X-rays showed good graft incorporation; however, sclerotic changes were seen. He was subjected to hip abductor strengthening exercises with gradual ambulation until he was able to achieve full weight bearing.

All authors declare that written informed consent was obtained from the patient for publication of this case report and accompanying images.

\section{DISCUSSION}

There have been numerous studies evaluating the risk factors of osteonecrosis but the etiology and pathogenesis remain unclear. The death of bone cells in osteonecrosis is the end point of the multifactorial pathology including ischemia, direct cellular toxicity, and alterations in the differentiation of mesenchymal stem cells that act individually or synergistically. ${ }^{2)}$ This case of NONFH reported as early detection proves to be a challenge in primary care.

There are limited published epidemiological data for NONFH avail-
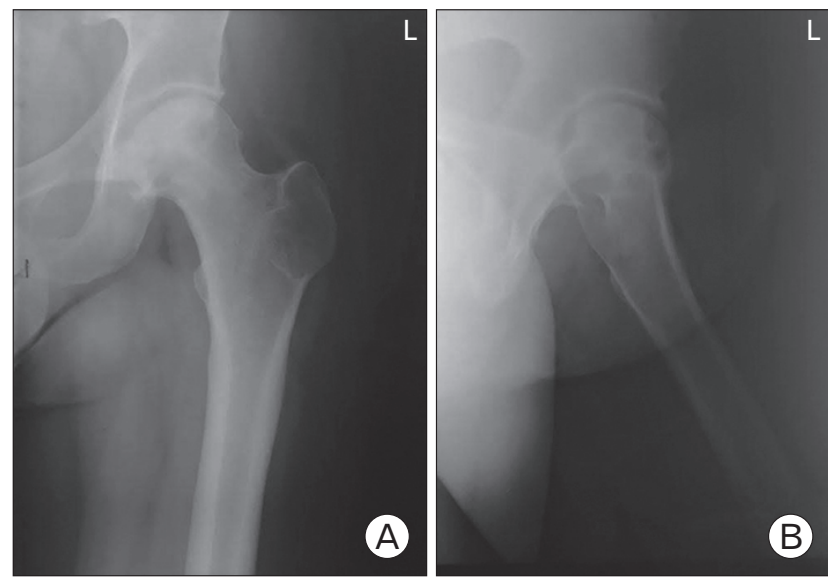

Figure 1. Left hip X-ray in anteroposterior (A) and oblique (B) views demonstrating abnormal sclerosis with mild flattening and irregularity of the femoral head contour. 

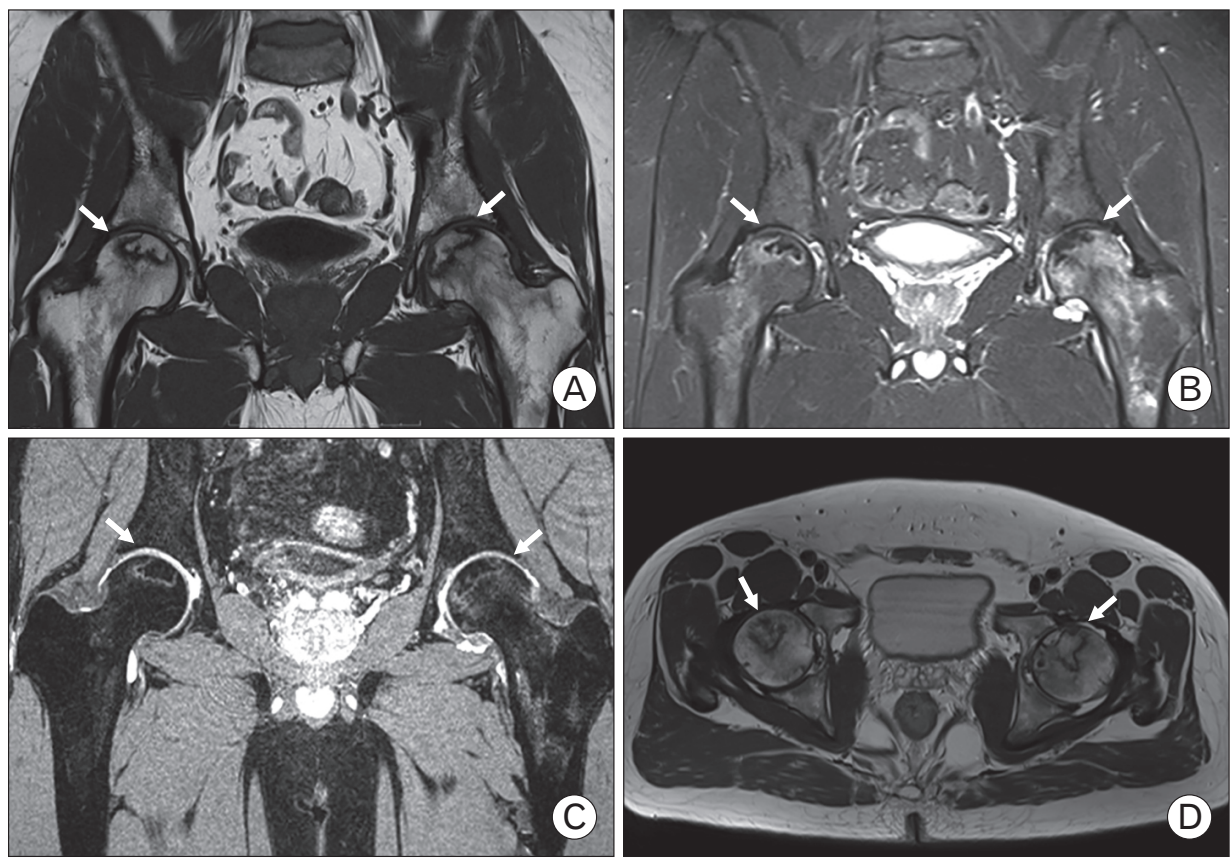

Figure 2. Magnetic resonance imaging images in the T1W coronal view (A), T1W - TIRM coronal view (B), T2 fat saturatedcoronal view (C), and proton density-axial view (D). Images demonstrating low signal serpiginous lines and subchondral edema of the femoral heads bilaterally (white arrows), consistent with avascular necrosis.

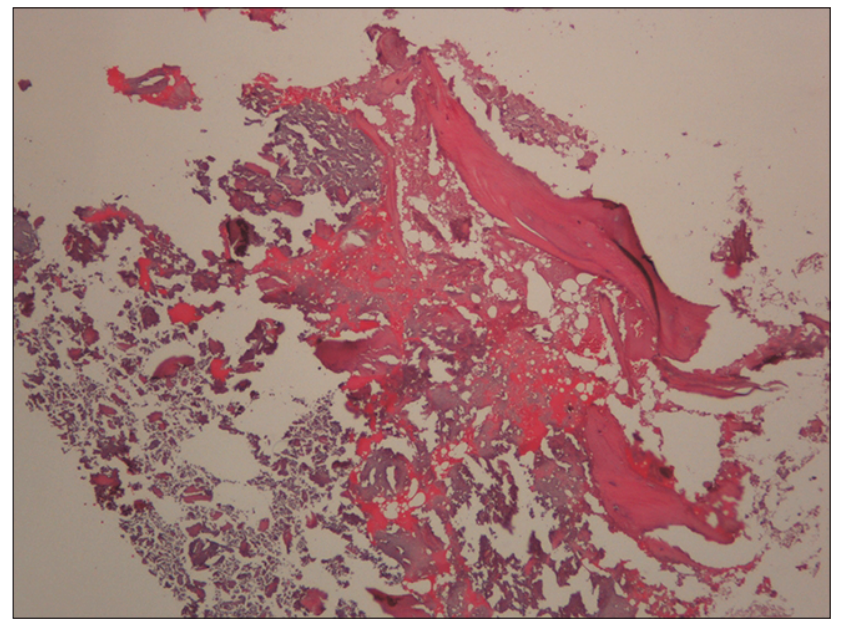

Figure 3. Histopathological examination of left femoral head biopsy showing necrotic bony tissue.

able for Malaysia or the Asian region as a whole. In the late 1990s, epidemiological studies showed that 10,000 to 20,000 new cases of NONFH were diagnosed in the United States each year. In Japan, with a population of 128 million, the annual incidence rate of NONFH is 1.91 per $100,000 .{ }^{3)}$ Meanwhile, in a nationally representative survey involving 30,030 respondents held in China, the overall prevalence of NONFH was reported as $0.725 \%$. ${ }^{4}$

The majority of NONFH cases have a secondary cause, as reported in a multicentric retrospective study among secondary care patients, which revealed the most common causes of diagnosed NONFH were alcohol use (30.7\%), steroid-induced (24.1\%), and idiopathic (28.8\%). Researchers are contemplating the possibility of a genetic predisposition, which proves irrelevant in this case. ${ }^{6)}$ Interestingly, in this case, there was no identifiable risk factors, thus, it seemed to be more of an idiopathic cause of NONFH.

This patient fits the demographic profile of most literature which states ONFH and NONFH is more common in males ${ }^{1,4,5)}$ within the age of 35 to 44 years old. ${ }^{4)}$ The patient presented with the typical presentation, which is hip pain, with varying severity of pain, which subsequently affects the patient's lifestyle and impairs their productivity. In this case, the patient presented with unilateral hip pain despite having bilateral NONFH. Studies have shown that bilateral NONFH can present as bilateral hip pain, unilateral hip pain or completely asymptomatic. ${ }^{6}$ Detailed assessment by history taking and a targeted physical examination could aid in ascertaining the specific etiology of hip pain. ${ }^{7)}$ This patient had complained of anterior left groin pain, which is the most common symptom for intra-articular pathologies such as NONFH. ${ }^{8)}$ It is important to identify some of the identifiable factors which were detected in this case, such as the presence of night pain, weight bearing pain, and the interference with daily activities, which allows a prompt case detection. ${ }^{8)}$ It is also pertinent to know of any childhood hip problems, trauma, level of physical activity, or sports played, although, in this case, these were insignificant.

Physical examination is generally sensitive; however, a systematic review found inadequate evidence to recommend many of the special examination tests. ${ }^{8)}$ In this highlighted case, a clinical diagnosis was made appropriately, especially after all other causes of pain and bony abnormalities were excluded and correlation from MRI was carried out. MRI without contrast is still held as the "gold standard" diagnostic modality, especially in the early stages of ONFH such as this."

Essentially, this case highlights the difficulties and barriers in making a diagnosis of NONFH in primary care due to the clinical presentation, absence of risk factors, and normal initial radiological findings, 
although clinical suspicion may arise after a period of symptoms not resolving. Besides, persistent hip pain in young adults such as this case is not normal; therefore, such cases should be investigated.

Conservative management such as rest, simple analgesia, or nonsteroidal anti-inflammatory and referral to physiotherapy are the typical management for hip pain. However, in ONFH, this conservative approach is useful for symptomatic treatment but ineffective in halting the progression of the disease. ${ }^{10)}$ Recent studies and research are leaning towards the aggressive operative treatment to halt the progression of the disease and to improve a patient's well-being. In this case, aggressive operative treatment maybe inevitable if disease progression is identified.

In conclusion, the pertinent points from this case are the importance of improving the diagnostic accuracy of NONFH by adequate history taking, targeted physical examination, and clinical acumen on identifiable symptoms. The presence of different types of pain, which include intermittent and recurrent left hip pain and low back pain radiating to the groin, should initiate further studies into the presentation of NONFH. Due to the non-specific presentation of NONFH and the likelihood of attributing the symptoms to other differential diagnoses, most cases are referred at a later stage. However, this case highlights that early investigation and referral for suspected NONFH are crucial to enable an effective approach to delay the progression of the disease.

\section{CONFLICT OF INTEREST}

No potential conflict of interest relevant to this article was reported.

\section{ORCID}

Nurul Nadia Baharum: https://orcid.org/0000-0001-8922-1296
Farnaza Ariffin: https://orcid.org/0000-0002-7755-9289

Mohammad Hanafiah: https://orcid.org/0000-0003-4791-497X

Shahrul Hisham Sulaiman: https://orcid.org/0000-0001-6125-9356

\section{REFERENCES}

1. Swarup I, Lee YY, Movilla P, Figgie MP. Common factors associated with osteonecrosis of the femoral head in young patients requiring total hip arthroplasty. Hip Int 2015;25:232-6.

2. Zalavras CG, Lieberman JR. Osteonecrosis of the femoral head: evaluation and treatment. J Am Acad Orthop Surg 2014;22:455-64.

3. Ikeuchi K, Hasegawa Y, Seki T, Takegami Y, Amano T, Ishiguro N. Epidemiology of nontraumatic osteonecrosis of the femoral head in Japan. Mod Rheumatol 2015;25:278-81.

4. Zhao DW, Yu M, Hu K, Wang W, Yang L, Wang BJ, et al. Prevalence of nontraumatic osteonecrosis of the femoral head and its associated risk factors in the Chinese population: results from a nationally representative survey. Chin Med J (Engl) 2015;128:2843-50.

5. Cui L, Zhuang Q, Lin J, Jin J, Zhang K, Cao L, et al. Multicentric epidemiologic study on six thousand three hundred and ninety five cases of femoral head osteonecrosis in China. Int Orthop 2016;40:267-76.

6. Mont MA, Zywiel MG, Marker DR, McGrath MS, Delanois RE. The natural history of untreated asymptomatic osteonecrosis of the femoral head: a systematic literature review. J Bone Joint Surg Am 2010;92: 2165-70.

7. Wilson JJ, Furukawa M. Evaluation of the patient with hip pain. Am Fam Physician 2014;89:27-34.

8. Dick AG, Houghton JM, Bankes MJK. An approach to hip pain in a young adult. BMJ 2018;361:k1086.

9. Etienne G, Mont MA, Ragland PS. The diagnosis and treatment of nontraumatic osteonecrosis of the femoral head. Instr Course Lect 2004;53:67-85.

10. Mont MA, Baumgarten KM, Rifai A, Bluemke DA, Jones LC, Hungerford DS. Atraumatic osteonecrosis of the knee. J Bone Joint Surg Am 2000;82:1279-90. 